IFT UAM/CSIC-2006-09

hep-th/0602285

\title{
Dynamical Higgs potentials with a landscape
}

\author{
J.L.F. BARBÓn AND C. HoYos \\ Instituto de Física Teórica IFT UAM/CSIC \\ C-XVI, UAM, Cantoblanco 28049. Madrid, Spain \\ jose.barbon@uam.es, c.hoyos@uam.es
}

\begin{abstract}
We consider one-loop effective potentials for adjoint Higgs fields that originate from flat holonomies in toroidal compactification of gauge theories. We show that such potentials are "landscape-like" for large gauge groups and generic non-supersymmetric matter representations. In particular, there is a large number of vacua with similar local properties, scanning a broad band of vacuum energies.
\end{abstract}

February 2006 


\section{Introduction}

In discussions of effective potentials defined over moduli spaces of string compactification (following the blueprint of [1]), one often emphasizes their very intricate nature. Working in the framework of low-energy effective actions (a notoriously dubious procedure for the task at hand [2]), the introduction of various trapped fluxes and other nonperturbative effects generates effective potentials with very rich structure, depicting an almost infinite variety of minima, maxima, valleys and rifts dubbed "the landscape" [3]. The com-

plicated features of these effective potentials are largely a consequence of the discreteness introduced by a large number of quantized fluxes and the variety of possible brane and singularity configurations [4]. In a sense, it comes as no surprise that a very complicated potential should be obtained from a system with a rich cocktail of degrees of freedom.

However, the scanning of low-energy couplings in a broad band of values, particularly the vacuum energy, is considered to be a general feature of landscape potentials. For example, it is incorporated as a crucial ingredient in field-theoretical toy examples [5, [0]. In this brief note we study one such field-theoretical toy model in which a landscape-like potential is generated dynamically from a higher dimensional gauge theory.

In this letter, we will refrain from any discussion of possible phenomenological applications, except for some brief comments at the end. Our main motivation is to show how a very complicated-looking potential can be generated out of relatively simple systems. In particular, we have few adjustable parameters affecting significantly the structure of the potentials, whose properties are largely a consequence of group theory effects.

We consider perturbative effective potentials for adjoint Higgs fields that are generated by dimensional reduction on a torus with flat connections. This type of geometrical construction is very well known, and has been widely studied since [7] (see [8] for a recent summary). The essential ingredient responsible for the "landscape" features is a parametrically large rank of the gauge group and the breaking of a global discrete symmetry that maps out vacua with similar local properties.

\section{Effective potentials for flat connections}

We begin by reviewing some standard facts about adjoint Higgs fields generated upon toroidal compactification. Let us consider a Yang-Mills model in a $(d+n)$-dimensional spacetime of the form $\mathbf{R}^{d} \times \mathbf{T}^{n}$, a product of a standard flat $d$-dimensional Minkowski 
spacetime and an internal torus of size $L$, which we take orthogonal for simplicity. The classical action

$$
S_{h}=\frac{1}{2 g_{h}^{2}} \int_{\mathbf{R}^{d} \times \mathbf{T}^{n}} \operatorname{tr}\left|F_{A B}\right|^{2}
$$

reduces, for distance scales much larger than $L$, to the effective system

$$
S=\frac{1}{2 g^{2}} \int_{\mathbf{R}^{d}} \operatorname{tr}\left(\left|F_{\mu \nu}\right|^{2}+\left|D_{\mu} \Phi_{a}\right|^{2}-\left|\left[\Phi_{a}, \Phi_{b}\right]\right|^{2}+\ldots\right)
$$

where we have split the $(d+n)$-dimensional indices $(A, B, \ldots)$ into $\mathbf{R}^{d}$ spacetime indices $(\mu, \nu, \ldots)$ and internal $\mathbf{T}^{n}$ indices $(a, b, \ldots)$. Each field $\Phi_{a}$ is an adjoint Higgs that originates in the Fourier modes of the gauge field components along the $n$-torus and the commutator term is their classical potential, coming from the "magnetic" energy density, $F_{a b} F^{a b}$, of field-strengths along the torus directions. The dots stand for higher order terms suppressed by powers of $E L$ in a low-energy expansion with $E \ll 1 / L$, and the classical mapping between high and low energy couplings is the standard Kaluza-Klein relation $g_{h}^{2}=L^{n} g^{2}$. Only the zero modes of the gauge field on $\mathbf{T}^{n}$ survive as massless adjoint Higgses in the effective theory below the gap $1 / L$. These are the flat connections on $\mathbf{T}^{n}$, defined by the vanishing of the magnetic energy $F_{a b}=0$. A gauge-covariant description of these degrees of freedom is given by topologically nontrivial Wilson lines wrapped around the noncontractible cycles of $\mathbf{T}^{n}$.

In general, the space of flat connections on $\mathbf{T}^{n}$ has connected components. These can be the result of specific group-theory properties (e.g. the nontrivial commuting triples for $n=3$ and particular gauge groups [9]), or be associated to topological structure of the gauge bundles, such as non-abelian electric and magnetic fluxes through the torus [10]. Each connected component of the moduli space admits a parametrization in terms of constant commuting gauge connections in some appropriate subgroup of the gauge group. Since most of our results apply to each connected component separately, in the following we focus on the particular case of the single connected component containing the identity Wilson lines, corresponding to bundles admiting periodic boundary conditions on the torus. A convenient parametrization of these flat connections is given by gauge fields that are constant on $\mathbf{T}^{n}$ and lie on the Cartan subalgebra of the gauge group. Modding out by the remaining gauge transformations one finds the moduli space

$$
\mathcal{M}=\frac{\left(\mathbf{T}_{\mathrm{C}}^{r}\right)^{n}}{W}
$$

where $r$ is the rank of the gauge group and $W$ is the Weyl group that permutes the eigenvalues of the Wilson lines. We refer to the torus $\left(\mathbf{T}_{\mathrm{C}}{ }^{r}\right)^{n}$ as the "toron valley", defined 
as the product of $n$ copies of $\mathbf{R}^{r} / 2 \pi \widetilde{\Lambda}_{\mathrm{r}}$, where $\widetilde{\Lambda}_{\mathrm{r}}$ is the dual of the root lattice. A set of coordinates on $\mathcal{M}$ is provided by the constant commuting connections

$$
L \vec{\Phi} \equiv \vec{\phi}=\sum_{i=1}^{r} \vec{\phi}_{i} H_{i}
$$

with $H_{i}$ a complete set of generators of the Cartan subalgebra. In these coordinates, the Cartan torus identification on $\vec{\phi}$ consists of translations by $4 \pi \alpha \vec{n}$, with $\alpha$ a root and $\vec{n} \in \mathbf{Z}^{n}$ a vector of integers. The Weyl group acts by reflections on the hyperplanes orthogonal to the roots, and makes $\mathcal{M}$ into an orbifold.

In the effective gauge theory on $\mathbf{R}^{d}$, the moduli space $\mathcal{M}$ is to be regarded as the target space for the Higgs fields. For $d>2$, it is also the space of their possible "expectation values" in a Coulomb phase, i.e. the gauge group left unbroken by the Higgs mechanism at a generic point on $\mathcal{M}$ is the maximal abelian subgroup. On submanifolds of higher codimension we have enhanced gauge symmetry, which remains completely unbroken at the origin of moduli space, together with the points on $\mathcal{M}$ that are obtained from $\vec{\phi}=0$ by the action of twisted gauge transformations [10]. These are transformations $U(\vec{z})$ that act by finite shifts of $\phi$, and are twisted around the nontrivial cycles of the torus as

$$
T_{a} U(\vec{z}) T_{a}^{-1}=z_{a} U(\vec{z})
$$

where $T_{a}$ is a translation by $L$ on the $a$-th direction of the torus and $z_{a}$ is a phase in the center of the gauge group. For example, for $S U(N)$ the center is $\mathbf{Z}_{N}$ and $z_{a}$ is given by any $N$-th root of unity. In general, if $N$ is the cardinal of the center, we find $N^{n}$ vacua that are locally identical to the unbroken vacuum labelled by $\vec{\phi}=0$.

\subsection{Effective potentials}

The low-energy effective action for fields, $\vec{\phi}$, parametrizing the Cartan torus (i.e. for those with vanishing classical potential $\left.\left[\Phi^{a}, \Phi^{b}\right]=0\right)$ includes an effective potential induced by integrating out all the high-energy degrees of freedom. Working in perturbation theory in the Yang-Mills coupling, we have a natural expansion parameter at the compactification scale $1 / L$. For a rank $N$ gauge group, it is given by the dimensionless 't Hooft coupling $\lambda=g^{2}(L) N L^{4-d}$, where $g^{2}(L)$ is the effective $d$-dimensional Yang-Mills coupling, renormalized at the scale $1 / L$. This parameter, defined at the matching scale $1 / L$ runs with energy according to

$$
\lambda_{\mathrm{eff}}(E)=\lambda(E L)^{d_{\mathrm{eff}}-4},
$$


with logarithmic running for $d_{\mathrm{eff}}=4$. The effective dimension is given by $d_{\mathrm{eff}}=d$ for $E L<1$, whereas $d_{\mathrm{eff}}=d+n$ for $E L>1$. Of course, perturbation theory necessarily breaks down at sufficiently high energies for $d+n>4$. The strong-coupling threshold is defined by $\lambda_{\text {eff }}\left(\Lambda_{\mathrm{UV}}\right)=1$, or

$$
\Lambda_{\mathrm{UV}}=\frac{\lambda^{-\frac{1}{d+n-4}}}{L}
$$

and defines the scale beyond which some ultraviolet (UV) completion must be provided. Effects of such UV physics decouple below the compactification scale $L$ as inverse powers of $\Lambda_{\mathrm{UV}} L$, or equivalently, as positive fractional powers of $\lambda$.

Keeping in mind these limitations of the perturbative approach, the one-loop effective potential can be defined by the gaussian functional integral over those gauge fields on $\mathbf{R}^{d} \times \mathbf{T}^{n}$ that are orthogonal to the flat connections. Since the flat connections $\vec{\phi}$ are not integrated over, they function as a background field and we may use the standard machinery of the background field gauge. We obtain for the one-loop effective potential

$$
\int_{\mathbf{R}^{d}} V_{\text {eff }}(\vec{\phi})=\frac{1}{2} \operatorname{Tr}_{\mathcal{V}^{T}}^{\prime} \log \left(-D_{M} D^{M}\right)_{\mathbf{R}^{d} \times \mathbf{T}^{n}}-[\vec{\phi}=0],
$$

where we have normalized the potential to vanish at the origin of moduli space $\vec{\phi}=0$ (here and in the following, the square bracket notation means that we subtract the previous expression with the appropriate vanishing parameter). The adjoint covariant derivative operator $D_{M}=\partial_{M}+i\left[A_{M}\right.$, ] is evaluated at the flat connection $A_{M}=(0, \vec{\Phi})$. The trace in (2.7) is taken over the space $\mathcal{V}^{T}$ of transverse vector fields on $\mathbf{R}^{d} \times \mathbf{T}^{n}$, and the prime means that zero modes of the operator $-D_{M} D^{M}$ are left out of the trace.

Examples of such potentials have been extensively studied in the literature. The cases $d=4$ have been considered repeatedly as models for Higgs sectors, starting with [7]. The cases $n=1$ are well-known from the literature on thermal field theory [11] (see the recent 12] for the case $d=0$ ), while the case $d=1$ appears in the small-volume expansion of gauge theories on tori [13, 14].1]

Factoring out the Lorentz and gauge group components of the functional trace we find

$$
V_{\text {eff }}(\vec{\phi})=(d+n-2) \sum_{\alpha \in \text { roots }} V_{0}(\alpha \cdot \vec{\phi}),
$$

where the dot stands for the Cartan inner product, $\alpha \cdot \vec{\phi}=\sum_{i=1}^{r} \alpha_{i} \vec{\phi}_{i}$. We have used the fact that the flat connection lies on the Cartan subalgebra, and the roots are the

1 In fact, the "landscape-like" properties reported below were originally found in the particular cases studied in [14]. 
eigenvalues of the Cartan generators. The purely scalar and abelian function, $V_{0}$, is given by

$$
\int_{\mathbf{R}^{d}} V_{0}(\vec{\xi})=\frac{1}{2} \operatorname{Tr} \log \left[-\partial_{\mu} \partial^{\mu}-\left(\vec{\partial}+i \frac{\vec{\xi}}{L}\right)^{2}\right]_{\mathbf{R}^{d} \times \mathbf{T}^{n}}-\frac{1}{2} \operatorname{Tr} \log \left[-\partial_{\mu} \partial^{\mu}-\vec{\partial}^{2}\right]_{\mathbf{R}^{d} \times \mathbf{T}^{n}}
$$

where the differential operators act now over scalar functions on $\mathbf{R}^{d} \times \mathbf{T}^{n}$, and the determinants are calculated in Euclidean signature. A proper time representation of this determinant is the following

$$
V_{0}(\vec{\xi})=-\frac{1}{2} \int_{0}^{\infty} \frac{d t}{t} \int \frac{d^{d} p}{(2 \pi)^{d}} \sum_{\vec{n} \in \mathbf{Z}^{n}} e^{-t\left(p^{2}+\left(\frac{2 \pi \vec{n}}{L}+\vec{\xi}\right)^{2}\right)}-[\vec{\xi}=0] .
$$

We can obtain a physical interpretation of the potential by integrating over the time component of momenta in $\mathbf{R}^{d}$,

$$
V_{\mathrm{eff}}(\vec{\phi})=\frac{1}{2}(d+n-2) \sum_{\alpha} \sum_{\vec{n} \in \mathbf{Z}^{n}} \int \frac{d \mathbf{p}}{(2 \pi)^{d-1}} \sqrt{\mathbf{p}^{2}+\left(\frac{\vec{n}}{L}+\frac{\alpha \cdot \vec{\phi}}{2 \pi L}\right)^{2}}-[\vec{\phi}=0],
$$

which has a straightforward interpretation as the sum of zero-point energies of all field oscillators for each of the $d+n-2$ spin degrees of freedom. Field oscillators along the compact torus suffer a frequency shift due to the constant holonomy $\vec{\phi}$. Therefore, the effective potential is of Coleman-Weinberg type.

As expected from its definition, the subtracted potential (2.10) gets no contribution from those modes in the Cartan subalgebra that are also constant throughout the torus, corresponding to the $\vec{n}=0, \alpha=0$ terms in the sums. Since these modes are precisely given by the background flat connections, $\vec{\phi}$, we can view (2.10) as a Wilsonian potential in which all non-zero modes plus non-abelian constant modes on the torus are integrated out. In fact, the constant non-abelian modes at $\vec{n}=0$ have an effective mass of order $|\alpha \cdot \vec{\phi}| / L$, and the Wilsonian separation of scales breaks down at points in $\mathcal{M}$ where the Higgs mechanism turns off, i.e. the orbifold points. The extra massless degrees of freedom induce infrared singularities in (2.10). In order to estimate these, we examine the $\vec{n}=0$ terms, with an ultraviolet cutoff in place,

$$
\Delta V(\vec{\xi})_{\mathrm{IR}}=-\frac{1}{(4 \pi)^{d / 2}} \int_{\epsilon^{2}}^{\infty} \frac{d t}{t} t^{-d / 2} e^{-t \vec{\xi}^{2} / L^{2}} .
$$

In this expression, it is plain that $|\vec{\xi}| / L$ plays the role of an infrared cutoff in the proper time integral, which is itself regularized in the ultraviolet by the length-squared $\epsilon^{2}$. We 
can choose $\epsilon=L$ to consider the contribution of non-abelian constant modes with energies ranging from the compactification scale $1 / L$ down to the infrared cutoff $|\vec{\xi}| / L$. Then we may write the previous expression in the form

$$
\Delta V(\vec{\xi})_{\mathrm{IR}}=-\frac{1}{(4 \pi)^{d / 2} L^{d}}\left(\vec{\xi}^{2}\right)^{d / 2} \Gamma\left(\vec{\xi}^{2},-d / 2\right)
$$

in terms of the incomplete Gamma function. For odd values of $d$ we find a non-analytic behaviour of branch-point type, $V_{0}(\vec{\xi}) \sim\left(\vec{\xi}^{2}\right)^{d / 2}$, with a logarithmic correction $V_{0}(\vec{\xi}) \sim$ $\left(\vec{\xi}^{2}\right)^{d / 2} \log \left(\vec{\xi}^{2}\right)$ for even values of $d$. In particular, we have a conical singularity for $d=1$, the case studied in [13,14]. At any rate, as long as $d>2$ the non-analytic terms are quantitatively subleading to the analytic terms of order $\vec{\xi}^{2}$, induced by the high-energy modes.

An alternative representation of the effective potential is obtained by first integrating over the full $\mathbf{R}^{d}$ momenta and subsequently performing a Poisson resummation on the discrete sum over the $\mathbf{Z}^{n}$ lattice,

$$
V_{0}(\vec{\xi})=-\frac{L^{n}}{2(4 \pi)^{\frac{d+n}{2}}} \int_{0}^{\infty} \frac{d t}{t} t^{-\frac{d+n}{2}} \sum_{\vec{\ell} \in \mathbf{Z}^{n}} e^{-\frac{L^{2}}{4 t} \vec{\ell}^{2}}\left(e^{i \vec{\ell} \vec{\xi}}-1\right) .
$$

Notice that the subtraction of the $\vec{\xi}=0$ term can be incorporated as a restriction in the discrete sum to non-vanishing $\mathbf{Z}^{n}$ vectors $\vec{\ell} \neq 0$. The discrete vectors $\vec{\ell}$ can be interpreted as "winding" numbers around the torus $\mathbf{T}^{n}$ of first-quantized paths of the particles being integrated out. Then, carrying out the proper-time integral we arrive at

$$
V_{0}(\vec{\xi})=\frac{\Gamma\left(\frac{d+n}{2}\right)}{\pi^{\frac{d+n}{2}}} \frac{1}{L^{d}} \sum_{\vec{\ell} \neq 0} \frac{\sin ^{2}\left(\frac{1}{2} \vec{\ell} \vec{\xi}\right)}{\left(\vec{\ell}^{2}\right)^{\frac{d+n}{2}}} .
$$

In this form the effective potential is seen to be positive definite, vanishing at the origin, with periodicity $\vec{\xi} \rightarrow \vec{\xi}+2 \pi \mathbf{Z}^{n}$, and reflection symmetry $\vec{\xi} \rightarrow-\vec{\xi}$. The maxima of $V_{0}(\vec{\xi})$ sit at $(\pi, \pi, \ldots, \pi)$ modulo $2 \pi \mathbf{Z}^{n}$, whereas the minima are the images of $\vec{\xi}=0$ under the periodicity symmetry.

An interesting property of the one-loop effective potential is its ultraviolet finiteness, after a $\vec{\phi}$-independent constant is appropriately subtracted. This is actually true to all orders in perturbation theory. The reason is that any counterterm of the $(d+n)$-dimensional gauge theory must be gauge-invariant and, as such, a polynomial in covariant derivatives and curvature field strengths. All those counterterms vanish on the space of flat connections, and therefore $V_{\text {eff }}$ cannot get UV divergences at any order in perturbation theory 
(see [15] for a recent analysis of counterterms in compactified gauge theories). The error made by extending the momentum integrals and sums beyond the UV threshold $\Lambda_{\mathrm{UV}}$ vanishes as $\Lambda_{\mathrm{UV}} L$ goes to infinity. Therefore, all corrections to $V_{\text {eff }}$ from the physics of the UV completion are controlled by the small parameter $\lambda \ll 1$.

\subsection{Generalizations}

The function $V_{0}(\vec{\xi})$ also determines the effective potential induced by integrating out matter fields in an arbitrary representation of the gauge group. For scalar fields or fermions in an irreducible representation $R$, we must substitute the number of on-shell degrees of freedom, $n+d-2$ in (2.10), by the corresponding number of on-shell degrees of freedom (polarization, charge, etc) in bosons or fermions, $\mathcal{N}_{R}$. In general, this number will also depend on whether the fields in question are massive or massless. At the same time, we must substitute the sum over roots (i.e. the weights of the adjoint representation) by a sum over weights in the representation $R$. At the end, we find

$$
V_{\text {eff }}(\vec{\phi})=\sum_{R}(-1)^{F_{R}} \mathcal{N}_{R} \sum_{\mu \in R} V_{M_{R}}(\mu \cdot \vec{\phi})
$$

where $(-1)^{F_{R}}$ is the fermion number of the representation. The potential function $V_{M}$ is the massive generalization of (2.9), obtained by the simple replacement $p^{2} \rightarrow p^{2}+M^{2}$. Carrying out the substitution, the representation (2.11) is simply modified by the insertion of $e^{-M^{2} t}$ in the proper-time integral. Finally, the final form of the potential (2.12) gets modulated as

$$
V_{M}(\vec{\xi})=\frac{2}{L^{d}}\left(\frac{M L}{2 \pi}\right)^{\frac{d+n}{2}} \sum_{\vec{\ell} \neq 0} \frac{K_{\frac{d+n}{2}}\left(M L \sqrt{\vec{\ell}^{2}}\right)}{\left(\vec{\ell}^{2}\right)^{\frac{d+n}{4}}} \sin ^{2}\left(\frac{1}{2} \vec{\ell} \vec{\xi}\right),
$$

where $K_{\nu}(x)$ is a modified Bessel function of the second kind. In the massless limit $M L \rightarrow 0$, we recover (2.12) through the limit $K_{\nu}(x \rightarrow 0) \sim \frac{1}{2} \Gamma(\nu)(2 / x)^{\nu}$. On the other hand, the asymptotics $K_{\nu}(x \gg 1) \sim e^{-x} \sqrt{\pi / 2 x}$ implies that the sum over winding modes is effectively cutoff at $|\vec{\ell}|<(M L)^{-1}$. In particular, for large masses on the compactification scale, $M L \gg 1$, the whole potential is suppressed by a factor of $\exp (-M L)$.

An interesting particular case concerns models with softly broken supersymmetry, i.e. broken by the mass splittings $M_{R}$. In this case, the total potential gets no contribution from the far UV regime, and the boson-fermion cancellation is complete when the nonsupersymmetric mass splittings are removed. In any case, notice that the mass terms do 
not have a large effect on the qualitative form of the potential function (2.14), which is dominated by small values of $|\vec{\ell}|$ even for the $M=0$ case. Therefore, the main effect of soft-breaking masses is a global quenching of the associated effective potentials, without major modifications of the qualitative features such as the symmetry properties (i.e. the location of the vacua with unbroken gauge symmetry).

A point of detail concerns the proper Wilsonian interpretation of the effective potentials induced by arbitrary matter representations. In the adjoint representation, all flat connections are zero modes of the adjoint covariant derivative operator. These zero modes were eliminated from the partition sums by the vacuum-energy subtraction. For generic matter representations, zero modes are located on submanifolds of zero measure in the moduli space $\mathcal{M}$. Therefore, we integrate out all matter degrees of freedom and let singularities of $V_{\text {eff }}$ appear on $\mathcal{M}$, much in the same fashion as the enhanced-symmetry singularities appear for the case of the adjoint representation.

Some further generalizations concern different spin structures for fermions on $\mathbf{T}^{n}$ (which amount to additive shifts of $\vec{\phi}$ in all formulae, see for example [16]), and twisted boundary conditions for the gauge bundles on $\mathbf{T}^{n}$. In this last case, the moduli space of flat connections is partially lifted (see [17] for a review). On the remaining moduli space the analysis is equivalent to what is described here.

\section{The Cartan-Weyl landscape}

The potential (2.13) has "landscape-like" features for generic representations. The overall features are dictated by the crystallographic nature of the compact moduli space $\mathcal{M}$, in particular the slightly different action of the Weyl group, $W$, depending on the representation under consideration. Associated to a given weight $\mu$ in a general representation, there are codimension $n$ hyperplanes on $\mathcal{M}$, defined by

$$
\mu \cdot \vec{\phi}=0 \bmod 2 \pi \mathbf{Z}^{n}
$$

that are fixed by $W$. Such Weyl hyperplanes host local minima of bosonic potentials (along transverse directions) and local maxima of fermionic potentials. Therefore, local minima (maxima) are found at intersections of Weyl hyperplanes, i.e. at the edges of the so-called Weyl chamber. The effective potential contributed by a representation $R$ has structure down to the overall scale determined by the size of the Weyl chamber, inversely proportional to the norm of the highest weight. Hence, very large representations induce 
potentials with short-distance scale on $\mathcal{M}$. The overall picture is that of an intricate pattern of rifts and valleys along the Weyl hyperplanes associated to different representations.

Fermionic contributions to the total effective potential look like "inverted" bosonic potentials. Hence, the singular locus of fermionic contributions is associated with local maxima rather than minima. Conversely, local minima of fermionic potentials are related to local maxima of bosonic potentials, and they could be smooth, just like the local maxima of the function (2.12). Such smooth local minima of the full potential will typically break the gauge symmetry in a complicated pattern.

An interesting question is the fate of vacua with "unbroken" gauge symmetry, corresponding to the zeros of the pure Yang-Mills effective potential. If the matter potential happens to be sufficiently smooth at those minima, its main effect is simply to lift the unbroken vacuum to a non-vanishing vacuum energy. The surprising fact is that these lifted vacua typically scan in a broad band of energies.

We now focus on $S U(N)$ gauge theories, which give rise to a very large group of central conjugations, i.e. $\left(\mathbf{Z}_{N}\right)^{n}$ with cardinal $N^{n}$. In this case, we have a large number of unbroken symmetry vacua on $\mathcal{M}$ and we can enquiry how are they lifted by the matter potentials. The zeros of the potential (2.10) are solutions of the equation

$$
\alpha \cdot \vec{\phi}_{0}=0 \bmod 2 \pi \mathbf{Z}^{n}
$$

for any root $\alpha$. The $S U(N)$ roots can be written as $\alpha^{(i j)}=\nu^{i}-\nu^{j}$, with $\nu^{i}$ the weights of the defining fundamental representation of $S U(N)$. These weights satisfy $\sum_{i=1}^{N} \nu^{i}=0$ and $\nu^{i} \cdot \nu^{j}=\left(N \delta^{i j}-1\right) / 2 N$. Hence, any $N-1$ of them form a basis of the Cartan subalgebra. This basis not being exactly orthogonal will prove a crucial fact in what follows. Expressing (3.1) in this basis, we obtain the full set of solutions parametrized as the discrete lattice

$$
\vec{\phi}_{0}=4 \pi \sum_{i} \vec{n}_{i} \nu^{i}, \quad \vec{n}_{i} \in \mathbf{Z}^{n}
$$

In the pure Yang-Mills theory, all these vacua with unbroken gauge symmetry are strict copies of the zero-Higgs vacuum $\vec{\phi}=0$. In fact, their occurrence is related to the action of the $\left(\mathbf{Z}_{N}\right)^{n}$ group of central conjugations, associated to large gauge transformations as in (2.5). Perturbatively, these vacua remain disconnected, since the tunneling amplitude across the potential barrier in $V_{\text {eff }}$ is nonperturbative in the gauge coupling.

In the presence of additional matter degrees of freedom, these vacua get lifted according to the value of

$$
\sum_{R}(-1)^{F_{R}} \mathcal{N}_{R} \sum_{\mu \in R} V_{M_{R}}\left(\mu \cdot \vec{\phi}_{0}\right) .
$$


Thus, for a given representation, we must evaluate

$$
V_{M}\left(4 \pi \sum_{i} \vec{n}_{i} \mu \cdot \nu^{i}\right)
$$

for all weights $\mu$. In general, any weight can be found in the weight lattice generated by the $\nu^{i}$. Therefore, the argument of the scalar function $V_{M}(\vec{\xi})$ will be determined by integer linear combinations of the scalar products

$$
4 \pi \nu^{i} \cdot \nu^{j}=2 \pi \delta^{i j}-\frac{2 \pi}{N}
$$

The term proportional to the Kronecker delta has no effect by the periodicity properties of (2.12), and we are left with a sum of terms of the form $V_{M}(2 \pi \vec{K} / N)$, where $\vec{K}$ is a $\mathbf{Z}^{n}$-valued vector defined modulo $N$. If $\vec{K}$ is of $O(1)$ in the large $N$ limit, then the full potential at this particular local vacuum is a sum of terms of $O\left(1 / N^{2}\right)$, since the potential can be considered quadratic near the origin (for $d>2$ ). On the other hand, if $\vec{K}=O(N)$, we have a sum of terms of $O(1)$. The final scaling of the potential depends in each case on the multiplicity from the sum over weights.

Our considerations refer only to the energy shift of the $N^{n}$ "unbroken" vacua in a $\left(\mathbf{Z}_{N}\right)^{n}$ representation. In principle, the matter potential can alter the local properties of the vacua beyond a simple shift of vacuum energy. If the slope of $V_{R}$ is large enough at $\vec{\phi}_{0}$, the local minimum can disappear. This is more likely to happen the larger is the representation contributing to $V_{\text {eff }}$, because the overall scale of the potential is proportional to the dimension of the representation. For this reason, stability of the unbroken vacua will require in general that the mass of matter representations be sufficiently large, so that the matter contributions are appropriately quenched by the $\exp (-M L)$ suppression factor. For $M L \gg 1$ and near the origin, we may approximate

$$
V_{M}(\vec{\xi}) \approx \Delta \vec{\xi}^{2}
$$

with

$$
\Delta \sim \frac{(M L)^{\frac{d+n-1}{2}}}{L^{d}} \exp (-M L) .
$$

For $d=2$ there is a correction by a logarithmic factor, $\log |\vec{\xi}|$, whereas for $d=1$ the leading approximation is linear in $|\vec{\xi}|$. In the following we consider the case $d>2$.

In general, the "unbroken" vacua are shifted by the matter contribution, so that the gauge symmetry at those vacua is actually broken. We can still refer to these vacua as 
"unbroken" in order to make explicit their origin in the $\mathbf{Z}_{N}$-vacua of the pure Yang-Mills theory. In fact, if the matter is even slightly heavier than the compactification scale, $M L>1$, we can have $\Delta \ll 1$ and the symmetry breaking effects (such as gauge boson masses) are suppressed by a factor of $O(\Delta)$.

\subsection{Examples}

To illustrate these points, we consider some examples. First, matter fields (fermionic or bosonic) in the fundamental representation of $S U(N)$. In this case, the weights are given directly by the $\nu^{i}$, so that the potential is proportional to

$$
\sum_{i} V_{M}\left(\frac{2 \pi}{N} \sum_{k} \vec{n}_{k}\right)=N V_{M}\left(\frac{2 \pi}{N} \sum_{k} \vec{n}_{k}\right) .
$$

For small values of the argument, we approximate the potential as in (3.3). Then, as $\sum_{k} \vec{n}_{k}$ varies from $O(1)$ to $O(N)$, the lifted local minima scan a band ranging from $O(1 / N)$ to $O(N)$, times the mass quenching factor $\Delta$, with spacings of order $\Delta / N$ at the bottom, and only of order $\Delta$ at the top of the band.

A second example is given by matter fields in the antisymmetric representation of $S U(N)$. In this case, weights are of the form $\nu^{i}+\nu^{j}$, with $i<j$. The matter potential evaluated at the lattice of unbroken symmetry points is proportional to

$$
\sum_{i<j} V_{M}\left(\frac{4 \pi}{N} \sum_{k} \vec{n}_{k}\right)=\frac{1}{2} N(N-1) V_{M}\left(\frac{4 \pi}{N} \sum_{k} \vec{n}_{k}\right) .
$$

The same reasoning as before shows that the lattice of $N^{n}$ points of unbroken $S U(N)$ symmetry is lifted to a band with typical (bottom) spacing of $O(1) \Delta$ and ranging up to $O\left(N^{2}\right) \Delta$ energies. If we consider the symmetric representation instead, we have to add the weights of the form $\mu=2 \nu^{i}$, introducing a finer structure of the type already described for the fundamental representation.

Our final example concerns the phenomenon of planar equivalence. It refers to the situation when nonsupersymmetric theories still show vanishing vacuum energy in the leading large $N$ approximation. In other words, when the total number of perturbative degrees of freedom, counting polarizations and various charges, including color, cancels among bosons and fermions. One can generate one such example by taking a supersymmetric theory, and replacing the gauginos in the adjoint representation by Dirac fermions in the symmetric or antisymmetric representations, in such a way that the counting of degrees of freedom 
differs from the supersymmetric one only at order $N$ (the so-called orientifold field theories [18], other examples can be found in [14]). For instance, in an orientifold-type model, the potential is proportional to

$$
V_{\text {eff }}(\vec{\phi})_{\text {ori }} \propto \sum_{i, j} V_{0}\left(\left(\nu^{i}-\nu^{j}\right) \cdot \vec{\phi}\right)-2 \sum_{i<j} V_{0}\left(\left(\nu^{i}+\nu^{j}\right) \cdot \vec{\phi}\right),
$$

up to terms of $O(N)$. In this case, the quenching effect of the matter mass is absent, so that the local vacuum structure of the full potential is significatively different from that of the pure Yang-Mills model. The interesting fact about this example is the vanishing of the averaged planar potential over the moduli space, i.e. the integral of $V_{\text {eff }}$ over the toron valley is only of $O(N)$, despite the fact that the generic scale of the potential is $O\left(N^{2}\right)$. To see this, we write the flat connection in the basis of simple roots: $\vec{\phi}=\sum_{l} \vec{c}_{l} \alpha_{s}^{l}$, with $\alpha_{s}^{l}=\nu^{l}-\nu^{l+1}, l=1, \ldots, N-1$ the simple roots. The orientifold potential takes the form $V_{\text {eff }}(\vec{\phi})_{\text {ori }}=\sum_{i, j}\left[V_{0}\left(\frac{1}{2}\left(\vec{c}_{i}-\vec{c}_{i-1}-\vec{c}_{j}+\vec{c}_{j-1}\right)\right)-V_{0}\left(\frac{1}{2}\left(\vec{c}_{i}-\vec{c}_{i-1}+\vec{c}_{j}-\vec{c}_{j-1}\right)\right)\right]+O(N)$.

In this expression, we have neglected terms of $O(N)$ coming from restrictions in range of the indices. When averaging over the toron valley, the coordinates $\vec{c}_{j}$ become dummy integration variables, and fermionic terms cancel out the bosonic ones by an appropriate change of variables. Similar reasoning shows that the squared potential is at most of $O\left(N^{3}\right)$. This means that potentials with the property of planar equivalence have no large "plateaus" at heights of $O\left(N^{2}\right)$. Instead, plateaus stay at $O(N)$ and all $O\left(N^{2}\right)$ peaks and valleys are relatively narrow.

\subsection{The general rules}

In general, the condition for the unbroken vacua to be lifted into a broad band of energies is that the global $\left(\mathbf{Z}_{N}\right)^{n}$ symmetry be broken by the matter representations. At the same time, the local stability properties of the pure Yang-Mills potential at those vacua should not be significantly upset, as is the case for matter potentials generated by relatively large masses on the compactification scale.

The misalignment responsible for the lifting of the vacua finds its origin in the slight non orthogonality of the basic weights $\nu^{i}$. If the expression of a given weight $\mu$ in terms

of the $\nu^{i}$ shows the same number of positive and negative signs modulo $N$, then the terms proportional to $2 \pi / N$ cancel out in the argument of the scalar potential $V_{M}(\vec{\xi})$. The coefficient of this term only depends on the representation, and not on the particular 
weight within it, because all those differ by a integer linear combination of roots, and the scalar product of roots with the $\nu^{i}$ leaves no $O(1 / N)$ residue.

If $\nu^{i}$ are the weights of the defining fundamental representation (the $N$ ), then $-\nu^{i}$ are weights of the conjugate representation (the $\bar{N}$ ). Any irreducible representation can be found in the decomposition of the tensor product of the $N$ and the $\bar{N}$ representations. Hence, the $(\bmod N)$ number of $\nu^{i}$ minus the number of $-\nu^{j}$ in the expression for $\mu$ is the $N$-ality of the representation, i.e. the character under the $\mathbf{Z}_{N}$ center of the group. At the end, the distribution of cosmological constants at the unbroken minima $\vec{\phi}_{0}=4 \pi \sum_{i} \vec{n}_{i} \nu^{i}$ is given by (up to an additive cosmological constant that is not determined by the potential)

$$
\Lambda\left(\vec{n}_{i}\right)=V_{\text {eff }}\left(\vec{\phi}_{0}\right)=\sum_{R} \mathcal{N}_{R}(-1)^{F_{R}} \operatorname{dim}(R) V_{M_{R}}\left(2 \pi \vec{K}_{R} / N\right),
$$

with the integer vector $\vec{K}$,

$$
\vec{K}_{R}=\eta_{R} \sum_{k} \vec{n}_{k}
$$

and $\eta_{R}$ the $N$-ality of the representation $R$.

Provided $M L$ is sufficiently large, each representation $R$ lifts the $N^{n}$ unbroken vacua into a band of width

$$
\Delta \Lambda=\operatorname{dim}(R) \Delta
$$

and level spacings in the range of

$$
\delta \Lambda=\operatorname{dim}(R)\left(\frac{\eta_{R}}{N}\right)^{2} \Delta
$$

at the bottom of the band. The previous results for the fundamental representation are obtained by setting $\operatorname{dim}(R)=N$ and $\eta_{R}=1$, whereas those for the symmetric and antisymmetric representations use $\operatorname{dim}(R)=\frac{1}{2} N(N \pm 1), \eta_{R}=2$. In general, it is found that a discretuum of vacuum energies is favoured by group-theory effects in the case of matter in the fundamental representation. For higher-dimension matter representations, a quasi-continuous band is still possible, but then it is entirely determined by the large mass hierarchy $M L \gg 1$.

The $N$-ality is defined modulo $N$, and any representation with vanishing $\eta_{R}$ fails to lift the local unbroken vacua. This does not mean that these vacua remain unaltered, since local properties, such as masses of particles, depend on the relative contribution of matter and gauge terms (for example, fermion contributions tend to turn the minimum into a local maximum). 


\section{Conclusions}

In this note we have exhibited a simple example of landscape-like potential generated dynamically in purely field-theoretical terms. It has few continuous adjustable parameters, owing its peculiar features to group-theory effects. The effective potential for theories whose matter sector breaks the global group of central conjugations shows a very intricate pattern of peaks and valleys. In particular, local vacua of unbroken $S U(N)$ symmetry get lifted across a band with potentially small level spacing. A degeneracy of $O\left(N^{n-1}\right)$ vacua remains, as a consequence of the hypercubic symmetry of $\mathbf{T}^{n}$. The vacuum splittings depend on the contribution of matter representations, and can be controlled by the quenching factor $\exp (-M L)$ induced by large masses. Hence, a large mass hierarchy between the matter representations and the compactification scale produces a finer band of cosmological constants.

The main limitation of these considerations is their perturbative nature. In interesting $d=4$ situations, the higher-dimensional Yang-Mills theory is necessarily ill-defined in the ultraviolet. A UV completion is needed at some threshold scale $\Lambda_{\mathrm{UV}} \gg 1 / L$. The accuracy of our description of the effective potentials depends on the ability to maintain a large hierarchy between this UV scale and the compactification scale. The dimensionless 't Hooft coupling normalized at the compactification scale $\lambda=g^{2}(L) N L^{4-d}$ controls our approximations, valid for $\lambda \ll 1$, with corrections suppressed by fractional powers of $\lambda$.

Given the relationship between higher-dimensional theories and quiver models, it would be interesting to check if analogous potentials with scanning properties can be derived in the framework of "deconstruction" [19]. Possible applications of these results to Kaluza-Klein model building will not be considered here. We simply note that any implementation of this construction in string models with higher-dimensional wrapped branes (the natural arena to reproduce these features) requires that the gauge spectrum on the world-volume remains non-supersymmetric much above the compactification scale. This means that such models must have high-scale supersymmetry breaking, at least in this sector. The additional requirement that $\lambda \ll 1$ implies that such potentials are likely to be useful only for the modeling of "hidden sectors" (for other recent "landscape" constructions in the open string sector, see [20]).

At any rate, for the "discretum" of vacuum energies to have any phenomenological interest, one would need to drastically reduce the energy gap between local vacua. The group-theoretical gaps are smaller for matter in the fundamental representation. Further suppression might be achieved by constraining the matter representations to be very 
massive, $M L \gg 1$. The global overall scale of the potential (both for matter and gauge contributions) can be reduced by placing the branes at the bottom of a long throat in the context of warped compactifications.

\section{Acknowledgements}

We thank Karl Landsteiner for useful comments. The work of C.H. was partially supported by FPU grant AP2002-0433 from MEC-Spain. The work of J.L.F.B. was partially supported by MCyT and FEDER under grant BFM2002-03881 and the European RTN network MRTN-CT-2004-005104. 


\section{References}

[1] S. Kachru, R. Kallosh, A. Linde and S. Trivedi, Phys. Rev. D68 (2003) 046005 [arXiv:hep-th/0301240.]

[2] T. Banks, [arXiv:hep-th/0412129.]

[3] L. Susskind, [arXiv:hep-th/0302219.]

[4] R. Bousso and J. Polchinski, J. High Energy Phys. 0006 (2000) 006 [arXiv:hepth/0004134.] M. Douglas, J. High Energy Phys. 0305 (2003) 046 [arXiv:hepth/0303194.]

[5] C. Escoda, M. Gómez-Reino and F. Quevedo, JHEP 0311 (2003) 065, [arXiv:hepth/0307160.] K. R. Dienes, E. Dudas and T. Gherghetta, Phys. Rev. D72 (2005) 026005 [arXiv:hep-th/0412185.]

[6] N. Arkani-Hamed, S. Dimopoulos and S. Kachru, [arXiv:hep-th/0501082.]

[7] Y. Hosotani, Phys. Lett. B126 (1983) 309.

[8] Y. Hosotani, [arXiv:hep-ph/0504272.]

[9] E. Witten, [arXiv:hep-th/9712028.]

[10] G. 't Hooft, Nucl. Phys. B153 (1979) 141.

[11] D. Gross, R. Pisarski and L. Yaffe, Rev. Mod. Phys. 53 (1981) 43.

[12] O. Aharony, J. Marsano, S. Minwalla, K. Papadodimas, M. Van Raamsdonk and T. Wiseman, J. High Energy Phys. 0601 (2006) 140 [arXiv:hep-th/0508077.]

[13] M. Lüscher, Nucl. Phys. B219 (1983) 233. P. van Baal, [arXiv:hep-th/0008206.]

[14] J.L.F. Barbón and C. Hoyos, [arXiv:hep-th/0507267.] C. Hoyos, [arXiv:hep-th/0512303.]

[15] E. Alvarez and A. F. Faedo, [arXiv:hep-th/0602150.]

[16] E. Alvarez and A. Nieto, Phys. Rev. D41 (1990) 3850.

[17] A. González-Arroyo, [arXiv:hep-th/9807108.]

[18] A. Armoni, M. Shifman and G. Veneziano, Nucl. Phys. B667 (2003) 170 [arXiv:hepth/0302163.]

[19] N. Arkani-Hamed, A.G. Cohen and H. Georgi, Phys. Rev. Lett. 86 (2001) 4757 [arXiv:hep-th/0104005.]

[20] J. Gomis, F. Marchesano and D. Mateos, J. High Energy Phys. 0511 (2005) 021 [arXiv:hep-th/0506179.] 